\title{
Hydrodynamics of Young Supernova Remnants and the Implications for their Gamma-ray emission
}

\section{Vikram Dwarkadas University of Chicago}

E-mail: vikrameoddjob.uchicago.edu

\begin{abstract}
Supernovae (SNe) are generally classified into Type I and Type II. Most SNe ( 80\%), including all the subtypes of Type II, and Type Ib/c, arise from the core-collapse of massive stars. During their lifetime, mass-loss from these stars considerably modifies the medium around the stars. When the stars explode as $\mathrm{SNe}$, the resulting shock wave will expand in this wind-modified medium. In contrast, Type Ia SNe will expand in a relatively uniform medium, but the dynamics are different from those of core-collapse $\mathrm{SNe}$. For young supernova remnants, the properties of the ejecta as well as the surrounding medium are important in determining the subsequent evolution of the SN shock wave, and the dynamics and kinematics of the remnant. This will influence the acceleration of particles at the SN shocks, and consequently affect the gamma-ray emission from the remnant.
\end{abstract}

Herein we discuss the expected properties, especially the density structure, of the medium around various types and sub-types of $\mathrm{SNe}$, as suggested by current stellar evolution models. Using analytic and semi-analytic models and numerical simulations, we investigate how these affect the kinematics of the SN shock waves, assess the impact this would have on the production of cosmic rays, and show how it influences the time-evolution of the hadronic gamma-ray emission from the remnant. In the case of SNRs evolving in a wind medium, the emission should reach a maximum early on, and thereafter decrease with time. For SNe in a constant density medium, the emission would be expected to increase with time upto the advent of the Sedov stage.

Cosmic Rays and the InterStellar Medium

24-27 June 2014

Montpellier, France

\footnotetext{
${ }^{*}$ Speaker.
} 


\section{Introduction}

Supernovae ( $\mathrm{SNe}$ ) are basically divided into two types - those that arise from the thermonuclear detonation of low mass white dwarf stars (the Type Ia $\mathrm{SNe}$ ) and those that arise from the corecollapse of massive stars, which include all the other types such as 1b, 1c, and all Type II SNe. The explosion that forms either type of SN results in the expansion of a fast shock wave into the surrounding medium. The expansion and evolution of this shock depends on the characteristics of both the ejected material from the SN (the SN 'ejecta') and the structure of the surrounding material. In particular the density structure of both the ejecta and ambient medium are important. In this paper we discuss the formation of the ambient medium around various types of $\mathrm{SNe}$ into which the shock wave evolves, the resultant evolution of the SN shocks within this medium, and the implications for the gamma-ray emission from the various types of SNe.

\section{Young Supernova Remnants}

We define young supernova remnants (SNRs) as those that are still in the ejecta-dominated stage, and have not yet reached the Sedov-Taylor phase. To reach the Sedov-Taylor phase, the SN shock front must sweep up an amount of mass several tens of times larger than the ejected mass [14], which may take hundreds of years, or even thousands of years for a SNR evolving in a low density medium, such as SN 1006. The expansion of a SNR in the ejecta dominated phase leads to the formation of a strong shock expanding into the ambient medium, and a reverse shock that expands back into the ejecta in a Lagrangian sense. The two are separated by a contact discontinuity that divides the shocked ejecta from the shocked ambient medium.

\subsection{Type Ia Supernovae}

Type Ia SNe are thought to arise from the deflagration or detonation of a white dwarf star, presumably in a binary system. Since the white dwarfs don't suffer from wind-driven mass-loss, we can assume, at least in the first approximation, that they do not modify the medium around them. Thus the medium can be taken to be the interstellar medium, with a constant density profile. It is of course possible that the companion star may modify the medium, at least close-in to the SN, which is a possibility that needs to be examined more thoroughly.

By comparing to the ejecta profiles obtained from Type Ia explosions, [14] showed that the best approximation to the ejecta density distribution is an exponential profile. This fits much better than a power-law distribution that has often been used by many authors. However the introduction of an exponential introduces an additional parameter, and thus a self-similar solution is no longer possible. As shown by [14], the radius of the forward shock given by this profile is comparable to that given by a power-law. However the radius of the reverse shock, and the density profile within the shocked region, is quite different.

The density and pressure profile of a Type Ia SNR expanding into a constant density medium is shown on the left hand side of Figure 1.

\subsection{Core-Collapse SN}

The evolution of core-collapse $\mathrm{SNe}$, and the nature of the medium surrounding them, is more complicated. Core-collapse $\mathrm{SNe}$ arise from massive stars $\left(\mathrm{M} \geq 8 \mathrm{M}_{\odot}\right)$. These stars lose mass 

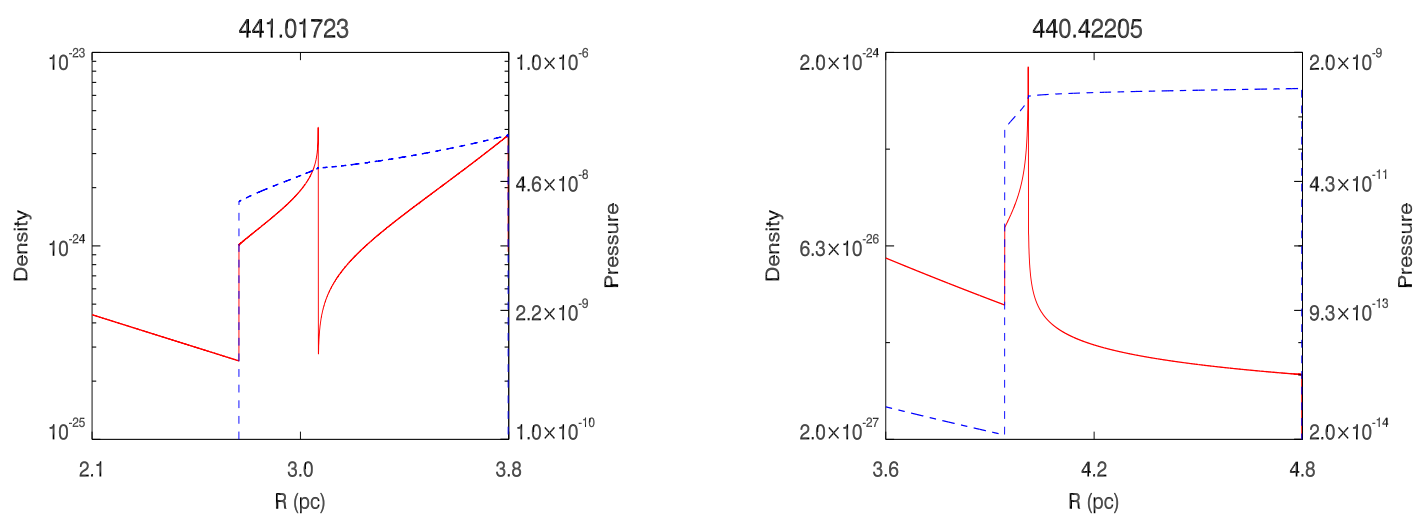

Figure 1: (Left) The density and pressure profiles for a Type Ia SN, described by an exponential profile, expanding into a constant density medium. (RHS) The profiles due to a Type II SN, described by a powerlaw profile, expanding into a wind-blown region. In both cases, the red line indicates the density (left axis scale), and blue the pressure (right axis scale). The radius is in parsecs, and time (top) is given in years. The grid is expanding, such that the outer edge of the grid lies just beyond the outer shock, which is therefore seen very close to the edge of the grid.

continually in the form of stellar winds, and for some stars via mass "eruptions". The amount of mass lost can be a substantial fraction of the stellar mass, and can modify the medium outside the star over a radius of several tens of parsecs [13], depending on the surrounding density.

The formation of the medium surrounding a massive star, due to the stellar wind mass-loss combined with ionization from the star, has been studied by many groups (e.g. [10, 20], and references within). In Figure 2 we show the evolution of the medium around a $40 \mathrm{M}_{\odot}$ star over the stellar lifetime $[12,10]$. In the main sequence phase (left), which occupies most of the star's lifetime, a wind bubble forms. Going outwards in radius, we see a freely expanding wind, windtermination shock, a low density region of shocked wind, an ionized region, a contact discontinuity separating the shocked surrounding medium from the bubble interior, and an outer shock, which is generally radiative. When the star leaves the main sequence to become a red supergiant (RSG), it blows a high mass-loss rate and low velocity wind, which creates a new pressure distribution and a high-density wind near the star. Eventually the star may lose its hydrogen, and sometimes He, envelope, becoming a Wolf-Rayet (W-R) star, and emitting a wind with a density that is a few times lower than in the RSG stage, but a velocity that is two orders of magnitude higher. This results in a W-R wind with a much higher momentum, capable of pushing the RSG wind out and mixing its contents in the bubble. At the same time, the density of the medium, which depends on the ratio of wind mass-loss rate to wind velocity, is considerably lower than in the RSG phase.

Although this is a simplified overview of one specific star, it illustrates some general considerations:

- Near a massive star, irrespective of which phase it is in, the density profile resembles that of a freely expanding wind medium. If the mass-loss rate and velocity are constant, this density will decrease as $\mathrm{r}^{-2}$. SNe from massive stars will initially expand within this wind region. 

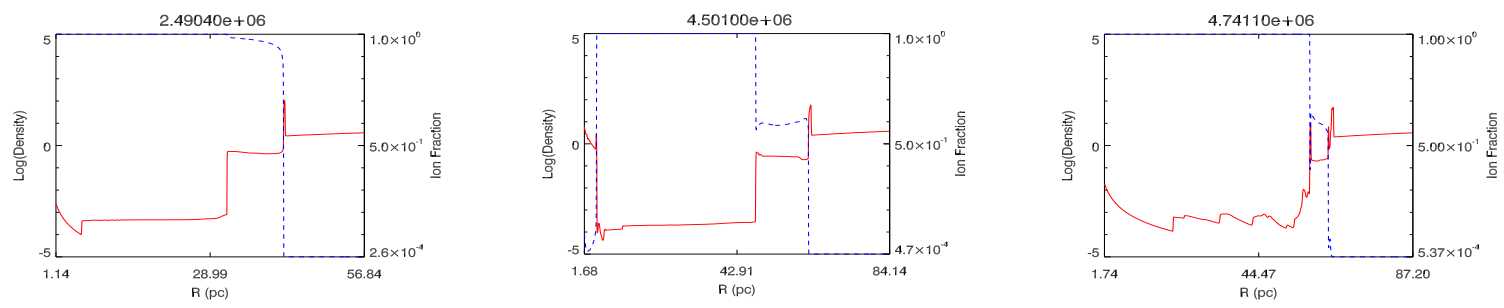

Figure 2: Evolution of the medium around a $40 \mathrm{M}_{\odot}$ star. The star goes through three stages, the main sequence phase (left), the red supergiant phase (middle) and the Wolf-Rayet phase (right). The red line displays the logarithm of the number density (scale on left); the blue is ionization fraction (scale on right). Radius is in parsecs, and time (top) is in years.

- For stars that explode to form SNe when in the RSG phase (Type IIP, IIL and possibly IIb SNe), the wind density around the star will be quite high, due to the high mass-loss rate and low wind velocity. Therefore these SNe will expand in a high density wind, although the density may fall after several hundred years (Figure 2, middle).

- For stars that explode to form SNe when in the W-R stage (Type Ib/c), the wind density is much lower than for IIPs (Figure 2, right). These stars are more compact than the RSGs as they have lost their $\mathrm{H}$ and, in some cases, He envelopes. The fast moving shock wave expands in a low density medium before colliding with the wind termination shock.

\section{Evolution of Young SNRs}

The evolution of a young SNR will depend on both its ejecta density profile and the nature and structure of the surrounding medium. As described above, Type Ia SNRs can be thought of as evolving, at least to zeroth order, in the constant density ISM. The crucial parameter is thus the density of the medium. Core-collapse $\mathrm{SNe}$ will evolve, at least initially, in the stellar wind, a medium of decreasing density. Over time however the density profile will change, which will directly affect the evolution.

The evolution of the supsersonic ejecta into the surrounding medium leads to a double-shocked structure, as described in $\S 2$ (Figure 1). The density structure of the shocked material within the two shocks depends on both the ejecta profile and the ambient medium, and will vary for various types of remnants expanding into different media. This will directly affect any emission process that is dependent on the density. Thus accurate calculations of the emission require that the hydrodynamics be properly calculated. If the cosmic-ray acceleration is efficient, and the energy expended in accelerating particles is high $(\geq 10 \%)$, then the back-reaction due to cosmic rays could affect the hydrodynamics itself, changing the shock structure and the relative positions of the forward shock, contact discontinuity and reverse shock $[2,16]$.

The SN shock waves interacting with the ambient medium can lead to a complicated evolution, especially for core-collapse SNe. The evolution has been studied by many authors (see [13, 12] and references therein). In the case of core-collapse SNe expanding in a low density wind-blown bubble (Figure 2, right), for example, the $\mathrm{SN}$ shock is first interacting with a freely expanding 


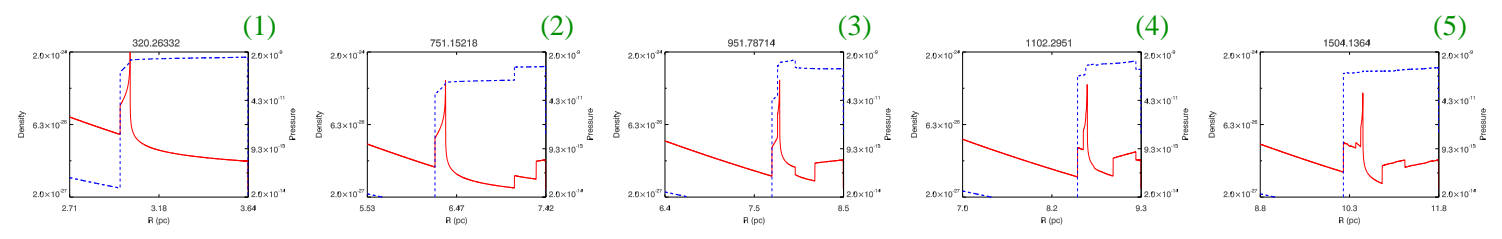

Figure 3: Evolution of a SN shock wave within the wind bubble created by a W-R star. The simulation focuses on the region in between the two shocks. In successive frames we show the SNR evolving in the freely expanding wind (1); colliding with the wind termination shock, giving a reflected shock that goes back into the ejecta; (3) the reflected shock crosses the contact discontinuity, sending a rarefaction wave into the shocked ambient medium; (4) and (5) the impact of the transient shock waves reduces as the complicated structure continues to expand outwards. The red line displays density (scale on left); the blue is pressure (scale on right). The radius is in parsecs, and time (top) is given in years.

wind, then collides with the wind-termination shock, and then expands into a more-or-less constant density medium (Figure 3), before eventually impacting the dense shell.

- As shown in [13] (Figure3), the impact of a strong shock on another shock wave or discontinuity leads to a transmitted shock into the medium and a reflected shock wave back into the ejecta. Thus, besides the SN shocks, there can be other shock waves in an inhomogeneous medium, which may at least temporarily accelerate particles to high energies. The observed spectrum is a composite of all of these various interactions, and should be appropriately computed.

- The density structure of the shocked material changes considerably as the SN moves from a wind medium to a constant density medium, which would affect the emission from the remnant.

- The shock velocity on impact with the wind termination shock is reduced, and then slowly increases again, which can affect the acceleration of particles.

It is important to realize (Figure 3) that even when a SNR is expanding in a wind-blown bubble, it need not be expanding in a freely expanding wind with a density profile that decreases as $\mathrm{r}^{-2}$, but could transition to a shocked wind with approximately constant density (with some fluctuations). The shock velocity and density structure will be modified. Furthermore, even when in the constant density region, the density profile may not resemble that it would have if it had originally started expanding in the constant density region [13]. This has implications for instance in modelling the $\gamma$-ray emission from SNR RXJ1713, assumed to arise within a low density wind bubble [15].

\section{Gamma-Ray emission}

Supernova shock waves can accelerate particles to high energies, presumably by the process of Diffusive Shock Acceleration [8]. The acceleration process is highly dependent on the magnetic field and magnetic turbulence ahead of the shock. This leads to a question, as yet unanswered, of whether the reverse shock is capable of accelerating particles, since the magnetic field in the SN ejecta is unknown. There is however no question that the forward shock can accelerate particles. The maximum energy of the particles depends on the magnitude of the magnetic field, and its orientation. Magnetic fields estimated from radio observations appear to far exceed the interstellar field, and it has been suggested that the back reaction of the cosmic rays themselves may amplify 
the field [1]. Although the actual mechanism remains unidentified, several instabilities have been identified that may serve to amplify the field. In a constant density medium, such as for Type Ia $\mathrm{SNe}$, the maximum energy of the accelerated particles will initially increase with time, although it will begin to decrease in most cases before the remnant has reached the Sedov stage $[11,18]$. In a wind medium, maximum energies on the order of a PeV could be reached in a few days [17], after which the energy of the particles would decrease with time.

The acceleration of protons to high energies, and their collision with stationary ambient protons, leads to hadronic emission via pion decay. For a SN expanding in a RSG wind (Figure 2, middle), the SN shock is first expanding in a high density RSG wind, but later will continue to expand in a low density medium created by the main sequence [19]. At some point the forward shock will begin to expand within the low density medium while the reverse shock will still be in the high density wind. If the reverse shock is accelerating particles to very high energies, then hadronic emission would predominate at the reverse shock, whereas accelerated electrons may be producing leptonic emission at the forward shock. The total $\gamma$-ray emission will be a combination of leptonic and hadronic processes, and not attributable to a single process. A combination of processes is possible in many other scenarios.

Using a simple analytic model for the hadronic emission [6], and the Chevalier self-similar solution [5] for the expansion of a young SNR into the surrounding medium, [9] calculated the evolution of the gamma-ray flux with time for a SNR expanding in a medium with a power-law profile. In the case of a wind medium with constant mass-loss parameters, the flux is given by:

$$
\begin{aligned}
F_{\gamma}\left(>E_{o}, t\right) & =\frac{3 q_{\gamma} B^{2} \xi\left(\kappa C_{1}\right) m^{3}}{2(5 m-m s-2) \beta \mu m_{p} d^{2}} t^{m-2} \\
& =\frac{3 q_{\gamma} \xi\left(\kappa C_{1}\right) m^{3}}{32 \pi^{2}(3 m-2) \beta \mu m_{p} d^{2}}\left[\frac{\dot{M}}{v_{w}}\right]^{2} t^{m-2}
\end{aligned}
$$

In the above equation, the contact discontinuity expands as $\mathrm{R}_{C D}=C_{1} t^{m}$, and the radius of the forward shock as $R_{s h}=\kappa R_{C D}=\kappa C_{1} t^{m}$. The velocity $v_{s h}=d R_{s h} / d t=m \kappa C_{1} t^{m-1}$ is therefore always decreasing with time. $q_{\gamma}$ is the $\gamma$-ray emissivity normalized to the cosmic-ray energy density (tabulated in [6]); $\xi$ denotes the fraction of the shock energy that is converted to cosmic rays; $d$ is the distance to the source; $\mu$ is the mean molecular weight and $m_{p}$ is the proton mass; $\dot{M}$ is the wind mass-loss rate and $v_{w}$ is the wind velocity; and $\beta \sim 0.3-0.5$ accounts for the fact that the volume of the shocked region from which the emission arises is smaller than the volume of the entire SNR.

The time dependence of the $\gamma$-ray flux is $\mathrm{t}^{m-2}$. Since for a wind-blown medium, $2 / 3<m \leq 1$ in the ejecta-dominated stage, this indicates that the flux is decreasing with time. Thus one would expect the flux to reach a maximum energy in a short period of time, and decrease from there on. The best time to observe $\mathrm{SN}$ in winds (essentially all core-collapse $\mathrm{SNe}$ ) would then be soon after explosion, when the flux is near maximum (see also [17]).

We can apply this to Cas A [9], assuming that it is expanding in a dense RSG wind, using the parameters in [3]. The Fermi best fit suggests that at most $2 \%$ of the total energy has gone into cosmic rays. If we assume $\xi=0.02$ then we get a flux $F_{\gamma_{C A S A}}(>100 \mathrm{MeV})=1.1 \times 10^{-8}$ that is comparable to the Fermi result. This suggests that a hadronic description may fit Cas A.

For a SNR expanding in a constant density medium, [9] found the flux to be given as: 

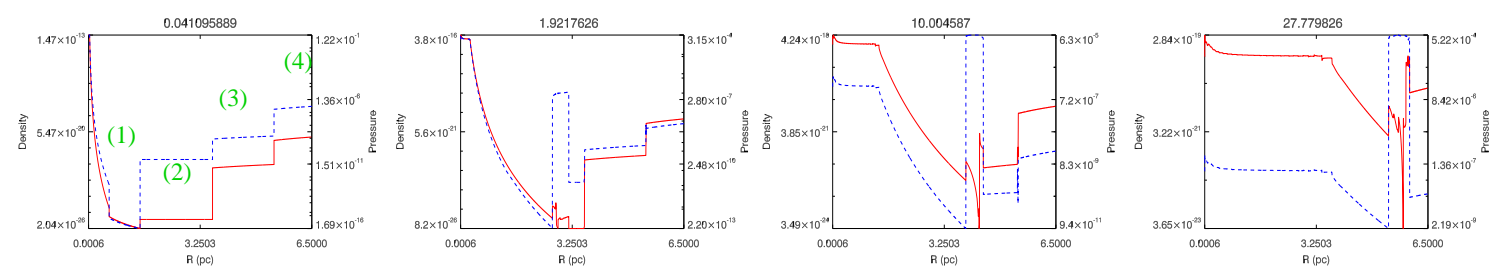

Figure 4: Evolution of the SN shock wave in the medium surrounding SN 1987A [7]. The medium consists of 4 regions (1) The freely expanding Blue Supergiant Wind (2) The Shocked Wind (3) The HII region and (4) The dense shell (equatorial ring), including the "fingers"responsible for the bright hotspots. The time evolution of the shock in regions (2), (3) and (4) is shown.

$$
F_{\gamma}\left(>E_{o}, t\right)=\frac{3 q_{\gamma} \xi\left(\kappa C_{1}\right)^{5} m^{3}}{6(5 m-2) \beta \mu m_{p} d^{2}} \rho_{a m}^{2} t^{5 m-2}
$$

For a SNR in a constant density medium, the parameter $m=2 / 5$ in the Sedov phase, and $m>2 / 5$ in the ejecta-dominated phase. Thus in a constant density medium the hadronic emission is increasing with time in the ejecta-dominated stage. Although this has been derived using a power-law profile, we expect a similar dependence for an exponential ejecta profile. This then suggests that core-collapse and Type Ia SNe show a different time-dependence in their hadronic emission, with the former decreasing with time and the latter increasing with time.

If a core-collapse SN were to expand in a constant density medium, its flux would increase with time. This is the expected case with SN 1987A, which is expanding into the high density HII region and dense shell created by the interaction of winds from the progenitor blue supergiant star [4]. The nature of the medium, and the evolution of the SN shock wave within this medium, is shown in Figure 4, using the parameters in [7]. Using equation 4.3 with the appropriate parameters [7], we find [9] (a) the flux is increasing with time, as expected, and (b) that in another 5-10 years, as the shock wave sweeps up more material, the flux will be large enough to be detectable potentially by the HESS array, and with high probability by the upcoming Cerenkov Telescope Array.

It is also useful to note the quadratic dependence on the density, as opposed to the linear dependence in [6]. While one power of density arises from the target mass, as in [6], the second power comes from the fact that the energy available to be extracted at the shock front is not a constant, but is in fact increasing with time. In the above $\xi$ is assumed to be constant; however it is quite possible that it could be a function of time.

Acknowledgements VVD's research on very high energy emission from young SNRs is partially supported by NASA Fermi grant NNX12A057G, and has benefited considerably from interaction with A. Marcowith, M. Pohl, M. Renaud, V. Tatischeff, and I. Telezhinsky. We are grateful to the FACCTS program for supporting travel to France, and fostering collaboration with University of Montpellier II. We thank A. Marcowith for hosting a very interesting and stimulating conference.

\section{References}

[1] A. R. Bell, and S. G. Lucek, Cosmic ray acceleration to very high energy through the non-linear amplification by cosmic rays of the seed magnetic field, MNRAS 321, (2001), 433 
[2] D. Castro, P. Slane, D. Patnaude and D. Ellison, The Impact of Efficient Particle Acceleration on the Evolution of Supernova Remnants in the Sedov-Taylor Phase, ApJ 734, (2011), 85

[3] R. A. Chevalier, and J. Oishi, Cassiopeia A and Its Clumpy Presupernova Wind, ApJ 593, (2003), 23

[4] R. A. Chevalier, and V. V. Dwarkadas, The Presupernova H II Region around SN 1987A, ApJL 452, (1995), L45

[5] R. A. Chevalier, Self-similar solutions for the interaction of stellar ejecta with an external medium, ApJ 258, (1982), 790

[6] L. O'C. Drury, F. A. Aharonian, and H. J. Voelk, The gamma-ray visibility of supernova remnants. A test of cosmic ray origin, $A \& A, \mathbf{2 8 7},(1994), 959$

[7] D. Dewey, V. V. Dwarkadas, F. Haberl, R. Sturm, and C. R. Canizares, Evolution and Hydrodynamics of the Very Broad X-Ray Line Emission in SN 1987A, ApJ 752, (2012), 103

[8] L. Oc. Drury, An introduction to the theory of diffusive shock acceleration of energetic particles in tenuous plasmas, RPP, 46, (1983), 973

[9] V. V. Dwarkadas, Exploring the $\gamma$-ray emissivity of young supernova remnants - I. Hadronic emission, MNRAS, 434, (2013), 3368

[10] V. V. Dwarkadas and D. Rosenberg, Simulated X-ray spectra from ionized wind-blown nebulae around massive stars, HEDP, 9, (2013), 226

[11] V. V. Dwarkadas, I. Telezhinsky, and M. Pohl, On the maximum energy and escape of accelerated particles in young supernova remnants, in proceedings of HIGH ENERGY GAMMA-RAY ASTRONOMY: 5th International Meeting, AIPC

[12] V. V. Dwarkadas, The Evolution of Supernovae in Circumstellar Wind Bubbles. II. Case of a Wolf-Rayet Star, ApJ, 667, (2007), 226

[13] V. V. Dwarkadas, The Evolution of Supernovae in Circumstellar Wind-Blown Bubbles. I. Introduction and One-Dimensional Calculations, ApJ, 630, (2005), 829

[14] V. V. Dwarkadas and R. Chevalier, Interaction of Type IA Supernovae with Their Surroundings, ApJ, 497, (1998), 807

[15] D. Ellison, P. Slane, D. Patnaude, and A. Bykov, Core-collapse Model of Broadband Emission from SNR RX J1713.7-3946 with Thermal X-Rays and Gamma Rays from Escaping Cosmic Rays, ApJ, 744, (2012), 39

[16] G. Ferrand, A. Decourchelle, and S. Safi-Harb, 3D Simulations of the Thermal X-Ray Emission from Young Supernova Remnants Including Efficient Particle Acceleration, ApJ, 760, (2012), 34

[17] A. Marcowith, M. Renaud, V. V. Dwarkadas, V. Tatischeff, Cosmic-ray acceleration and gamma-ray signals from radio supernovae, in proceedings of Cosmic Ray Origin beyond the standard models, [arXiv:1409.3670]

[18] K. M. Schure, and A. R. Bell, Cosmic ray acceleration in young supernova remnants, MNRAS, $\mathbf{4 3 5}$, 2013, 1174

[19] I. Telezhinsky, V. V. Dwarkadas, and M. Pohl, Acceleration of cosmic rays by young core-collapse supernova remnants, $A \& A, \mathbf{5 4 1},(2012), 153$

[20] J. A. Toala, and S. J. Arthur, Radiation-hydrodynamic Models of the Evolving Circumstellar Medium around Massive Stars, ApJ, 737, (2011), 100 